\section{Fenótipos corporais na adolescência e a maturação sexual}

\author{
Body phenotypes in adolescence and sexual \\ maturation
}

\section{Fenotipos corporales en la adolescencia y madurez sexual}

Jéssica Cumpian-Silva 1

Ana Elisa Madalena Rinaldi 2

Camila Medeiros da Silva Mazzeti 1

Wolney Lisboa Conde 1

doi: 10.1590/0102-311X00057217

\section{Resumo}

Nosso objetivo é descrever fenótipos corporais estimados por técnica multivariada para avaliação do estado nutricional na adolescência, durante o processo de maturação sexual. A amostra foi composta por 833 adolescentes escolares de 10 a 15 anos selecionados por amostragem complexa em Piracicaba, São Paulo, Brasil. Os fenótipos corporais foram definidos por análise de componentes principais (ACP) a partir de dados antropométricos (massa corporal, altura, dobras cutâneas e circunferência da cintura), de composição corporal (ângulo de fase medido por impedância bioelétrica), bioquímicos (triglicerídeos, glicose, razão colesterol total/LDL, hemoglobina) e de maturação sexual (autoclassificação dos estágios de pelos pubianos e mamas ou gônadas). Os fenótipos corporais foram: $F 1_{\text {adiposidade, }}$ caracterizado pela associação positiva com as variáveis dobras cutâneas, massa corporal e circunferência da cintura; $F 2_{\text {puberdade, }}$ caracterizado pela associação positiva com estágios de pelos pubianos, mama em meninas ou gônada em meninos, altura e idade; $F 3_{\text {bioquimico }}$ caracterizado pela associação positiva com colesterol, triglicerídeos e glicose; e F $4_{\text {muscular }}$ caracterizado pela associação positiva com ângulo de fase, hemoglobina e negativa com glicose. Somente

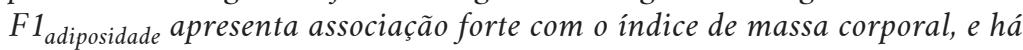
independência entre $F 1_{\text {adiposidade }}$ F $2_{\text {puberdade. Nossos resultados ressaltam a }}$ independência apresentada entre parâmetros bioquímicos, de antropometria, de composição corporal e de maturação sexual. A proposta de fenótipos corporais poderá embasar cálculo de escores da probabilidade de estar obeso a partir das variáveis antropométricas e superar a ambiguidade na utilização da massa corporal.

Maturidade Sexual; Biomarcadores; Composição Corporal; Adolescente

\section{Correspondência}

J. Cumpian-Silva

Faculdade de Saúde Pública, Universidade de São Paulo. Av. Dr. Arnaldo 715, São Paulo, SP 01246-904, Brasil. jesscumpian@gmail.com

1 Faculdade de Saúde Pública, Universidade de São Paulo, São Paulo, Brasil.

2 Faculdade de Medicina, Universidade Federal de Uberlândia, Uberlândia, Brasil. 


\section{Introdução}

A análise do estado nutricional de adolescentes é usualmente realizada com base no índice de massa corporal (IMC), sem levar em consideração as etapas da maturação sexual. Ao longo da adolescência, e em especial durante a maturação sexual, a massa corporal apresenta expressiva variação em sua composição ${ }^{1}$. Essa variabilidade tem potencial para alterar a associação convencional entre aumento da massa corporal e adiposidade. A elevada variabilidade das frações da composição corporal observada nessa etapa do ciclo vital leva alguns autores a fundamentar e se posicionar de modo diverso com relação à necessidade de ajuste prévio da maturação sexual para avaliação nutricional de adolescentes 1,2 .

Os principais argumentos a favor do ajuste pela maturação sexual são: (1) em adolescentes com puberdade precoce, existe aumento da espessura das dobras cutâneas e da massa corporal, portanto o ajuste contribuiria para a correta classificação do estado nutricional, levando em consideração as modificações corporais precoces causadas pela alteração da maturação sexual; (2) o IMC varia significativamente entre os estágios da maturação sexual, destacadamente no sexo feminino; além disso, algumas medidas antropométricas como altura, circunferência da cintura e massa corporal variam entre os estágios de maturação sexual; (3) existe associação positiva entre a maturação sexual precoce e o excesso de massa corporal em adolescentes, com evidências mais claras para o sexo feminino; e (4) alguns autores sugerem que a magnitude do efeito da maturação sexual sobre a composição corporal seja expressiva, a ponto de impactar a avaliação nutricional $3,4,5$.

Os principais argumentos contrários ao ajuste pela maturação sexual são: (1) o crescimento e o desenvolvimento puberal são processos altamente regulados biologicamente e, nesse sentido, a variabilidade intraestágio de maturação não é intensa a ponto de modificar a classificação do estado nutricional entre os estágios; (2) a obesidade ou excesso de massa corporal estão associados à precocidade da maturação sexual, mas não às diferenciações entre os estágios; (3) a tendência secular do IMC não explica isoladamente a tendência secular da precocidade da maturação sexual; e (4) diferenças inferiores a dois anos entre a idade média da maturação sexual da população estudada e da população de referência não introduzem distorções na avaliação do estado nutricional dos indivíduos analisados 1,6,7.

A simultaneidade não uniforme do aumento da massa corporal e do ganho de altura como função da idade ao longo da adolescência é um desafio para o processo de avaliação do estado nutricional, especialmente para a avaliação nutricional baseada em índices unidimensionais (o IMC, por exemplo), já que torna mais complexa a interpretação das mudanças antropométricas e da composição corporal ao longo da maturação sexual 8,9.

A questão-chave para análise das diferenças antropométricas parece ser os fatores determinantes da variação da massa corporal ao longo dos estágios de maturação sexual e entre estes. Tanto o IMC quanto a idade de início da maturação sexual mostram efeitos de tendência secular, o que adiciona complexidade ao processo de avaliação nutricional de adolescentes e consideráveis dificuldades para a comparação entre as evidências apresentadas contra ou a favor do ajuste da avaliação nutricional pela maturação sexual 1,7. Além disso, a massa corporal é reconhecida como um expressivo modificador dos parâmetros e indicadores da maturação sexual 10,11,12,13.

A complexidade das modificações da composição corporal durante a puberdade não é adequadamente abordada com o uso dos indicadores nutricionais tradicionais, uma vez que isolam fatores como antropometria, composição corporal ou marcadores bioquímicos 7,14. Uma alternativa a essa limitação é o uso de análise multivariada para a definição de fenótipos corporais. O fenótipo é conceituado como o somatório das especificidades que caracterizam um indivíduo 15. Operacionalmente, o fenótipo corporal foi expresso pela análise simultânea de variáveis das dimensões antropométricas, da composição corporal e dos marcadores bioquímicos em modelo multivariado.

A análise multivariada do estado nutricional permite integrar as múltiplas interações entre o fenômeno da maturação sexual e o processo do crescimento físico, bem como a análise de seus determinantes antropométricos, da composição corporal ou dos parâmetros bioquímicos sobre o estado nutricional dos adolescentes. Assim, a análise multivariada permite explorar a natureza das divergências encontradas na literatura sobre o ajuste do IMC por meio dos estágios de maturação sexual.

Desta forma, nosso objetivo é descrever fenótipos corporais a partir de variáveis que representem parâmetros antropométricos, de composição corporal, de maturação sexual e de marcadores 
bioquímicos, estimados por técnica multivariada, e apresentar uma alternativa para a avaliação do estado nutricional durante a adolescência.

\section{Métodos}

\section{Bases de dados}

Nesta análise, os dados são provenientes de adolescentes de 10 a 15 anos, residentes na cidade de Piracicaba, São Paulo ( $\mathrm{N}=833,57 \%$ do sexo feminino). Os dados do presente estudo são oriundos do desenho transversal do inquérito intitulado Determinantes do Risco de Obesidade entre Adolescentes a Partir de Inquérito de Escolares com Amostra Mista: Transversal e Longitudinal (IAP-SP). O IAP-SP corresponde à terceira onda de inquéritos realizados na cidade de Piracicaba com adolescentes escolares. Sinteticamente, seu processo amostral se deu por amostragem probabilística das escolas, utilizando dois critérios de estratificação: geográfico (centro e bairros periféricos) e tipo de administração escolar (público e particular) 16,17. O projeto IAP-SP foi aprovado pelo Comitê de Ética na Pesquisa da Faculdade de Saúde Pública, Universidade de São Paulo (processo no 154865, de 27 de novembro de 2012).

\section{Avaliação do estado nutricional}

A descrição dos fenótipos corporais foi feita por análise multivariada, a partir das variáveis de três dimensões mensuradas:

(a) Antropometria e composição corporal: massa corporal, altura, dobras cutâneas tricipital e subescapular, circunferência da cintura e ângulo de fase. Todas as medidas antropométricas foram realizadas segundo os procedimentos recomendados pelo Manual de Referência para Padronização de Medidas Antropométricas, em duplicata e expressas pela média dos dois valores 18,19. O IMC foi calculado dividindo-se a massa corporal $(\mathrm{kg})$ pela altura ao quadrado $\left(\mathrm{m}^{2}\right) 20$. O ângulo de fase foi estimado por impedância bioelétrica (BIA) (modelo 0358T, RJL Systems, Clinton Township, Estados Unidos) 21,22. Os procedimentos foram realizados segundo a recomendação do manual do fabricante. $\mathrm{O}$ ângulo de fase foi calculado como arco tangente da razão entre reactância e resistência. Todas as variáveis utilizadas são contínuas.

(b) Maturação sexual: a referência utilizada para a avaliação da maturação sexual foi aquela proposta por Tanner, que está baseada nos estágios da distribuição de pelos pubianos para ambos os sexos e nos estágios do desenvolvimento das mama em meninas ou da gônada em meninos 23,24 . A avaliação foi realizada por autoclassificação, a partir de pranchas ilustrativas contendo as cinco fases de cada um dos critérios (pelo pubiano, gônada e mama) 25. Todos os adolescentes autoclassificaram-se em um dos cinco estágios de maturação em cada critério. A autoclassificação assemelha-se à avaliação direta por profissionais de saúde em estudos comparativos ${ }^{26}$. Além disso, a Organização Mundial da Saúde (OMS) recomenda o uso da autoclassificação em estudos populacionais por diminuir custos e ser mais apropriada para superar barreiras psicossociais $27,28,29$.

(c) Marcadores bioquímicos: as amostras de sangue foram coletadas após jejum de 12 horas, segundo protocolo Técnicas para Coleta de Sangue do Ministério da Saúde 30. Foram dosados os valores de triglicerídeos $(\mathrm{mg} / \mathrm{dL}$ ), glicose (glicemia de jejum $\mathrm{mg} / \mathrm{dL}$ ), hemoglobina glicada $(\mathrm{g} / \mathrm{dL})$ e a razão colesterol total/LDL (mg/dL) 31,32 .

\section{Análise dos dados}

A análise de componentes principais (ACP) foi utilizada para definir os fenótipos corporais (variáveis latentes) a partir do conjunto de medidas demográficas, antropométricas, bioquímicas, da composição corporal e da maturação sexual. Foram retidos os componentes com eigenvalues superiores a 0,7 e, nestes, os eigenvectors superiores a $|0,2|$. A adequação da amostra em relação ao grau de correlação parcial entre as variáveis foi estimada pelo teste Kaiser-Meyer-Olkin (KMO) 33,34.

Os componentes gerados a partir da ACP foram nomeados como fenótipo corporal, seguido do número que designa a ordem de variabilidade explicada e um termo que expressassem as variáveis de 
interesse para avaliação do estado nutricional contidas no fenótipo. O primeiro fenótipo, por exemplo, foi representado por $\mathrm{F} 1_{\text {adiposidade. }}$

A associação entre cada fenótipo corporal e o IMC foi medida pelo estimador de Pearson (r). A análise de variância (ANOVA) e testes de comparação múltipla foram realizados para verificar a presença de diferenças étnicas entre os estágios de desenvolvimento puberal, entre os grupos étnicos, entre os fenótipos corporais e entre as variáveis antropométricas, bioquímicas e da composição corporal. O nível de significância adotado foi de 0,05 .

A ACP foi replicada separadamente em ambos os sexos para verificar possíveis diferenças na formação dos fenótipos corporais. A análise indicou não haver diferenças entre os fenótipos formados no conjunto dos dados e aqueles formados separadamente por sexo. Os resultados a seguir descrevem a análise realizada no conjunto dos dados, sem separação por sexo.

\section{Resultados}

Os valores médios de idade e das variáveis representativas do estado nutricional aumentaram conforme a evolução dos estágios de maturação sexual, segundo os cinco estágios de pelos pubianos e gônadas ou mamas (Tabela 1). Essa tendência de aumento reflete a evolução e a linearidade no desenvolvimento puberal dos adolescentes.

As principais variáveis antropométricas, bioquímicas, de composição corporal ou de maturação sexual não mostraram diferenças estatisticamente significantes entre os agrupamentos étnicos da amostra em estimativa realizada por análise de variância.

Foram retidos os quatro primeiros componentes (F1 a F4) que responderam por 69,3\% da variabilidade total. $\mathrm{O}$ teste $\mathrm{KMO}$ foi de 0,78 , valor que classifica a matriz multivariada como de alta consistência interna.

$\mathrm{O} F 1_{\text {adiposidade }}$ caracterizou-se por variáveis que expressam gordura (dobras do tríceps e subescapular) e volume corporal (massa corporal e circunferência da cintura). Esse fenótipo apresentou associação negativa com as variáveis de maturação sexual (-0,09 para pelo pubiano e -0,05 para gônada e mama). O F2 ${ }_{\text {puberdade }}$ caracterizou-se pelas variáveis da maturação sexual e pelas variáveis altura e idade, que marcam o eixo cronológico na adolescência. $\mathrm{O} F 3_{\text {bioquímico }}$ caracterizou-se por marcadores metabólicos (colesterol, triglicerídeos e glicose), ligados ao acúmulo de gordura corporal. Nesse fenótipo, as variáveis de maturação sexual apresentaram carga fatorial baixa ou negativa. O F4 muscular apresentou associação positiva com as variáveis ângulo de fase e hemoglobina, e associação negativa com glicose. Esse fenótipo identifica marcadores metabólicos relacionados à massa magra. $\mathrm{O}$ F4 muscular apresentou carga fatorial negativa para as variáveis de maturação sexual (Tabela 2).

A Figura 1 apresenta a dispersão dos escores dos $\mathrm{F} 1_{\text {adiposidade }} \mathrm{F} 2_{\text {puberdade, gerados a partir da }}$ ACP, segundo os valores do IMC. O F1 adiposidade apresenta associação linear e $\mathrm{r}$ forte com o IMC. Os demais fenótipos apresentam associações menos lineares e $\mathrm{r}$ de menor valor com o IMC (Figuras $2 \mathrm{e}$ 3). O padrão das associações analisadas indica que o tipo de correlação entre o F $1_{\text {adiposidade }}$ o IMC é diferente das correlações com os outros fenótipos.

A carga fatorial expressa a magnitude e o sentido da associação de cada variável com os fenótipos extraídos dos dados. As variáveis da maturação sexual apresentaram cargas fatoriais elevadas somente para $\mathrm{F} 2$ puberdade, o qual foi caracterizado majoritariamente por variáveis que refletem crescimento linear e expansão de todos os compartimentos corporais. Além disso, o F2 puberdade apresentou carga negativa para as variáveis que expressam gordura corporal (dobras cutâneas). O F $3_{\text {bioquímico }}$ e F 4 muscular apresentaram carga fatorial baixa ou negativa para maturação sexual e gordura corporal (Tabela 2).

As variáveis altura e idade mostraram-se mais significativamente associadas apenas com o F 2 puberdade. Nos outros fenótipos, a associação foi virtualmente nula para idade, e fraca ou nula para altura.

\section{Discussão}

A análise multivariada permitiu a estimativa de quatro perfis distintos quanto às variáveis associadas à avaliação do estado nutricional e, em dois deles, características opostas quanto à composição 
Tabela 1

Distribuição de médias e desvio padrão (DP) da idade e das variáveis representativas do estado nutricional, estratificada por estágios de maturação sexual (pelos pubianos e gônodas ou mamas) de adolescentes de ambos os sexos. Piracicaba, São Paulo, Brasil, 2012.

\begin{tabular}{|c|c|c|c|c|c|c|c|c|c|c|}
\hline \multirow[t]{2}{*}{ Variáveis representativas do estado nutricional } & \multicolumn{5}{|c|}{ Pelos pubianos } & \multicolumn{5}{|c|}{$\begin{array}{c}\text { Gônada ou mama } \\
\text { Média [DP] }\end{array}$} \\
\hline & 1 & 2 & 3 & 4 & 5 & 1 & 2 & 3 & 4 & 5 \\
\hline \multicolumn{11}{|l|}{ Meninos } \\
\hline \multirow[t]{2}{*}{ Altura } & 147,9 & 148,2 & 152,6 & 161,7 & 164,5 & 146,3 & 148,8 & 153,2 & 162,4 & 165,2 \\
\hline & {$[12,5]$} & {$[10,5]$} & {$[8,5]$} & {$[9,8]$} & {$[8,7]$} & {$[10,4]$} & {$[10,3]$} & {$[9,0]$} & {$[10,0]$} & {$[9,1]$} \\
\hline \multirow[t]{2}{*}{ Massa corporal } & 46,4 & 46,2 & 47,7 & 52,1 & 61,5 & 46,2 & 46,0 & 48,3 & 56,3 & 57,9 \\
\hline & {$[12,7]$} & {$[17,5]$} & {$[12,9]$} & {$[12,8]$} & {$[15,0]$} & {$[19,5]$} & {$[15,1]$} & {$[13,1]$} & {$[14,1]$} & {$[12,7]$} \\
\hline \multirow[t]{2}{*}{ Índice de massa corporal } & 21,0 & 20,6 & 20,2 & 20,6 & 22,6 & 21,0 & 20,4 & 20,4 & 21,2 & 21,1 \\
\hline & {$[4,5]$} & {$[5,7]$} & {$[4,5]$} & {$[3,8]$} & {$[4,8]$} & {$[5,8]$} & {$[4,9]$} & {$[4,5]$} & {$[4,6]$} & {$[3,8]$} \\
\hline \multirow[t]{2}{*}{ Dobra cutânea do tríceps } & 16,0 & 15,5 & 15,4 & 14,3 & 17,1 & 15,4 & 16,7 & 14,7 & 15,3 & 13,8 \\
\hline & {$[7,8]$} & {$[8,8]$} & {$[12,7]$} & {$[6,7]$} & {$[8,2]$} & {$[8,7]$} & {$[14,6]$} & {$[6,9]$} & {$[7,8]$} & {$[6,2]$} \\
\hline \multirow[t]{2}{*}{ Dobra cutânea subescapular } & 12,4 & 13,6 & 11,5 & 11,8 & 15,2 & 13,1 & 12,8 & 12,0 & 12,9 & 12,3 \\
\hline & {$[6,8]$} & {$[10,3]$} & {$[7,1]$} & {$[6,7]$} & {$[8,6]$} & {$[10,9]$} & {$[8,4]$} & {$[7,4]$} & {$[7,8]$} & {$[7,2]$} \\
\hline \multirow[t]{2}{*}{ Circunferência da cintura } & 72,1 & 71,7 & 70,9 & 72,9 & 77,0 & 72,6 & 71,7 & 70,8 & 74,4 & 73,9 \\
\hline & {$[11,4]$} & {$[14,5]$} & {$[10,5]$} & {$[9,8]$} & {$[12,4]$} & {$[15,2]$} & {$[13,0]$} & {$[10,4]$} & {$[11,4]$} & {$[9,4]$} \\
\hline \multirow[t]{2}{*}{ Ângulo de fase } & 6,6 & 6,4 & 6,6 & 6,6 & 7,1 & 6,7 & 6,2 & 6,6 & 6,7 & 6,9 \\
\hline & {$[1,1]$} & {$[1,2]$} & {$[1,4]$} & {$[1,0]$} & {$[1,1]$} & {$[1,2]$} & {$[0,8]$} & {$[1,5]$} & {$[1,1]$} & {$[0,7]$} \\
\hline \multirow[t]{2}{*}{ Razão colesterol total/LDL } & 3,7 & 3,9 & 3,7 & 3,8 & 3,8 & 3,6 & 3,9 & 3,8 & 3,7 & 3,7 \\
\hline & {$[0,9]$} & {$[0,9]$} & {$[0,8]$} & {$[0,8]$} & {$[0,7]$} & {$[0,8]$} & {$[0,9]$} & {$[0,8]$} & {$[0,8]$} & {$[0,6]$} \\
\hline \multirow[t]{2}{*}{ Hemoglobina } & 14,0 & 14,3 & 14,0 & 14,4 & 14,1 & 14,0 & 14,2 & 14,2 & 14,3 & 14,2 \\
\hline & {$[0,6]$} & {$[1,1]$} & {$[1,1]$} & {$[1,3]$} & {$[1,1]$} & {$[1,3]$} & {$[1,1]$} & {$[1,1]$} & {$[1,3]$} & {$[1,1]$} \\
\hline \multirow[t]{2}{*}{ Triglicerídeos } & 82,9 & 73,5 & 65,8 & 60,6 & 72,3 & 65,1 & 76,9 & 68,5 & 61,8 & 61,9 \\
\hline & {$[104,2]$} & {$[32,7]$} & {$[29,7]$} & {$[23,8]$} & {$[40,0]$} & {$[31,5]$} & {$[63,5]$} & {$[31,0]$} & {$[30,5]$} & {$[18,5]$} \\
\hline \multirow[t]{2}{*}{ Glicose } & 90,7 & 91 & 91,1 & 92,5 & 93,9 & 87,5 & 89,6 & 92,0 & 92,9 & 95,1 \\
\hline & {$[12,4]$} & {$[8,2]$} & {$[8,8]$} & {$[9,4]$} & {$[7,8]$} & {$[11,6]$} & {$[10,0]$} & {$[8,1]$} & {$[8,7]$} & {$[8,6]$} \\
\hline \multirow[t]{2}{*}{ Idade } & 11,7 & 12,0 & 12,7 & 13,4 & 13,9 & 12,1 & 12,0 & 12,7 & 13,5 & 13,9 \\
\hline & {$[1,5]$} & {$[1,0]$} & {$[1,1]$} & {$[1,1]$} & {$[0,9]$} & {$[1,4]$} & {$[1,2]$} & {$[1,1]$} & {$[1,0]$} & {$[0,9]$} \\
\hline \multicolumn{11}{|l|}{ Meninas } \\
\hline Altura & 149,4 & 151,4 & 156,2 & 158,3 & 157,1 & 144,8 & 149,5 & 155,5 & 158,5 & 157,2 \\
\hline & {$[11,3]$} & {$[8,5]$} & {$[6,6]$} & {$[6,3]$} & {$[7,3]$} & {$[10,0]$} & {$[7,8]$} & {$[7,5]$} & {$[6,2]$} & {$[5,6]$} \\
\hline Massa corporal & 44,3 & 50,8 & 51,2 & 54,5 & 52,6 & 39,3 & 44,3 & 50,4 & 55,7 & 57,7 \\
\hline & {$[13,5]$} & {$[18,6]$} & {$[11,3]$} & {$[12,9]$} & {$[9,5]$} & {$[9,6]$} & {$[11,5]$} & {$[12,5]$} & {$[13,2]$} & {$[14,9]$} \\
\hline Índice de massa corporal & 19,5 & 21,4 & 20,9 & 21,7 & 21,3 & 18,6 & 19,6 & 20,8 & 21,9 & 23,3 \\
\hline & {$[4,2]$} & {$[5,3]$} & {$[4,1]$} & {$[4,5]$} & {$[3,3]$} & {$[3,8]$} & {$[4,0]$} & {$[4,7]$} & {$[3,9]$} & {$[5,6]$} \\
\hline Dobra cutânea do tríceps & 15,7 & 18,4 & 18,0 & 19,2 & 19,3 & 14,4 & 17,6 & 17,2 & 19,7 & 21,3 \\
\hline & {$[5,9]$} & {$[7,5]$} & {$[10,3]$} & {$[7,3]$} & {$[6,9]$} & {$[6,4]$} & {$[13,7]$} & {$[6,4]$} & {$[7,2]$} & {$[6,8]$} \\
\hline Dobra cutânea subescapular & 11,6 & 14,2 & 14,6 & 15,3 & 13,8 & 10,1 & 13,5 & 13,4 & 15,5 & 17,6 \\
\hline & {$[4,9]$} & {$[8,0]$} & {$[6,9]$} & {$[7,0]$} & {$[5,2]$} & {$[4,4]$} & {$[7,3]$} & {$[6,0]$} & {$[6,9]$} & {$[9,4]$} \\
\hline Circunferência da cintura & 67,7 & 71,3 & 71,3 & 72,9 & 71,1 & 67,6 & 69,0 & 70,1 & 73,2 & 76,5 \\
\hline & {$[10,2]$} & {$[11,2]$} & {$[9,5]$} & {$[10,0]$} & {$[7,7]$} & {$[11,3]$} & {$[10,8]$} & {$[9,3]$} & {$[9,0]$} & {$[12,3]$} \\
\hline Ângulo de fase & 6,4 & 6,2 & 6,2 & 6,2 & 6,1 & 6,0 & 6,1 & 6,1 & 6,4 & 6,2 \\
\hline & {$[1,4]$} & {$[0,9]$} & {$[1,0]$} & {$[0,9]$} & {$[1,2]$} & {$[1,3]$} & {$[1,0]$} & {$[1,1]$} & {$[0,9]$} & {$[1,3]$} \\
\hline Razão colesterol total/LDL & 4,0 & 3,9 & 3,7 & 3,8 & 3,7 & 3,8 & 3,7 & 4,0 & 3,7 & 3,9 \\
\hline & {$[1,5]$} & {$[0,8]$} & {$[0,9]$} & {$[0,8]$} & {$[0,9]$} & {$[1,0]$} & {$[1,0]$} & {$[0,8]$} & {$[0,8]$} & {$[1,0]$} \\
\hline Hemoglobina & 14,4 & 13,9 & 13,9 & 14,0 & 14,0 & 13,8 & 14,0 & 14,0 & 14,0 & 14,0 \\
\hline & {$[1,2]$} & {$[1,2]$} & {$[1,0]$} & {$[1,2]$} & {$[1,0]$} & {$[1,0]$} & {$[1,2]$} & {$[1,0]$} & {$[1,1]$} & {$[1,4]$} \\
\hline Triglicerídeos & 80,5 & 86,4 & 79,1 & 79,5 & 72,6 & 70,1 & 83,8 & 84,4 & 75,5 & 83,9 \\
\hline & {$[67,0]$} & {$[41,7]$} & {$[42,1]$} & {$[37,0]$} & {$[36,1]$} & {$[35,3]$} & {$[37,9]$} & {$[49,7]$} & {$[34,9]$} & {$[54,9]$} \\
\hline Glicose & 85,8 & 90,2 & 89,0 & 90,5 & 89,8 & 85,6 & 89,9 & 90,5 & 89,3 & 89,7 \\
\hline & {$[8,1]$} & {$[9,4]$} & {$[9,2]$} & {$[8,4]$} & {$[7,9]$} & {$[8,3]$} & {$[9,7]$} & {$[8,8]$} & {$[8,5]$} & {$[8,8]$} \\
\hline Idade & 12,6 & 12,4 & 12,8 & 13,4 & 13,5 & 11,6 & 12,0 & 12,8 & 13,5 & 13,7 \\
\hline & {$[1,5]$} & {$[1,2]$} & {$[1,1]$} & {$[1,1]$} & {$[1,2]$} & {$[1,0]$} & {$[0,9]$} & {$[1,1]$} & {$[1,1]$} & {$[1,0]$} \\
\hline
\end{tabular}




\section{Tabela 2}

Cargas fatoriais dos quatro fenótipos corporais de adolescentes (10-15 anos) escolares, estimados em análise de componentes principais (ACP). Piracicaba, São Paulo, Brasil, 2012.

\begin{tabular}{|c|c|c|c|c|}
\hline \multirow[t]{2}{*}{ Variáveis representativas do estado nutricional } & \multicolumn{4}{|c|}{ Cargas fatoriais } \\
\hline & F1 adiposidade & $F 2_{\text {puberdade }}$ & $\mathrm{F}_{\text {bioquímico }}$ & F4 muscular \\
\hline Altura & 0,07 & 0,47 & $-0,04$ & 0,07 \\
\hline Massa corporal & 0,43 & 0,19 & 0,03 & 0,04 \\
\hline Dobra cutânea triciptal & 0,50 & $-0,08$ & $-0,06$ & $-0,09$ \\
\hline Dobra cutânea subescapular & 0,52 & $-0,06$ & $-0,02$ & $-0,01$ \\
\hline Circunferência da cintura & 0,48 & 0,02 & 0,08 & 0,00 \\
\hline Ângulo de fase & 0,13 & 0,08 & 0,09 & 0,32 \\
\hline Pelos pubianos & $-0,09$ & 0,47 & $-0,00$ & $-0,13$ \\
\hline Gônada ou mama & $-0,05$ & 0,48 & 0,01 & $-0,03$ \\
\hline Razão colesterol total/LDL & 0,01 & 0,01 & 0,64 & 0,19 \\
\hline Hemoglobina & $-0,08$ & 0,09 & 0,12 & 0,63 \\
\hline Triglicerídeo & $-0,00$ & $-0,04$ & 0,70 & $-0,16$ \\
\hline Glicose & $-0,03$ & 0,14 & 0,20 & $-0,61$ \\
\hline Idade & 0,03 & 0,46 & $-0,06$ & 0,03 \\
\hline \multicolumn{5}{|l|}{ Estatísticas } \\
\hline Autovalores & 3,30 & 2,60 & 1,39 & 1,06 \\
\hline \% da variância & 25,5 & 20,1 & 10,7 & 8,2 \\
\hline \% da variância acumulada & 25,5 & 45,5 & 56,2 & 64,5 \\
\hline KMO & \multicolumn{4}{|c|}{0,78} \\
\hline
\end{tabular}

KMO: Kaiser-Meyer-Olkin.

corporal. Em nossas análises, dois padrões de associação se destacaram. O primeiro deles caracteriza um fenótipo com maior volume corporal e elevada adiposidade, sem associação com maturação sexual ( $\mathrm{F} 1_{\text {adiposidade }}$ ) o segundo caracteriza um fenótipo de maturação sexual associada à expansão da massa óssea (traduzida pela altura) e presença do vetor cronológico (traduzido pela idade - F2 $2_{\text {puber- }}$ dade). Os dois últimos fenótipos corporais ( $\mathrm{F} 3_{\text {bioquímico }}$ e $\left.\mathrm{F} 4_{\text {muscular }}\right)$ apresentaram distinção de marcadores metabólicos: o F $3_{\text {bioquímico }}$ mostrou-se associado a possíveis alterações metabólicas atreladas à gordura corporal e o F4 muscular, associado à massa muscular.

Nossos resultados indicaram que, para esta análise, a etnia não se mostrou um fator determinante para a diferença dos fenótipos corporais entre os estágios de maturação sexual descritos por pelos pubianos e gônada ou mama.

A associação positiva da massa corporal com os dois primeiros fenótipos reflete dois processos distintos da composição corporal possíveis de serem analisados mais discriminadamente pela análise multivariada. Embora em ambos os fenótipos ocorra expansão da massa corporal, essa expansão só se faz com aumento da adiposidade no primeiro fenótipo ( $\mathrm{F} 1_{\text {adiposidade }}$. Desta forma, as principais controvérsias entre considerar ou não a maturação sexual na avaliação do estado nutricional de adolescentes pode recair na forma de interpretação de indicadores de avaliação nutricional que consideram a massa corporal para diagnóstico ${ }^{35}$. Durante a adolescência, a composição corporal, em especial o tecido adiposo, varia em função das idades de início e término da puberdade e da velocidade do crescimento físico 1. O diagnóstico baseado na massa corporal, por essa razão, pode expressar aumentos da massa corporal provenientes de processos fisiológicos diferentes, com composições distintas 36,37. É provável que a proposta de ajuste prévio do IMC segundo os estágios de maturação sexual para interpretação do estado nutricional seja derivada dessa ausência de discriminação dos processos fisiológicos subjacentes ao aumento da massa corporal. Adicionalmente, os processos biológicos do desenvolvimento físico nessa etapa do ciclo vital podem ser descritos por variáveis antropométricas, em análises multivariadas, e não apenas por aquelas tradicionalmente utilizadas na avaliação da maturação sexual. 
Figura 1

Correlação dos $\mathrm{F} 1_{\text {adiposidade }}$ e $\mathrm{F} 2_{\text {puberdade, }}$ definidos por análise de componentes principais (ACP) com o índice de massa corporal (IMC), segundo sexo. Piracicaba, São Paulo, Brasil, 2012.

1a) Masculino



1b) Feminino

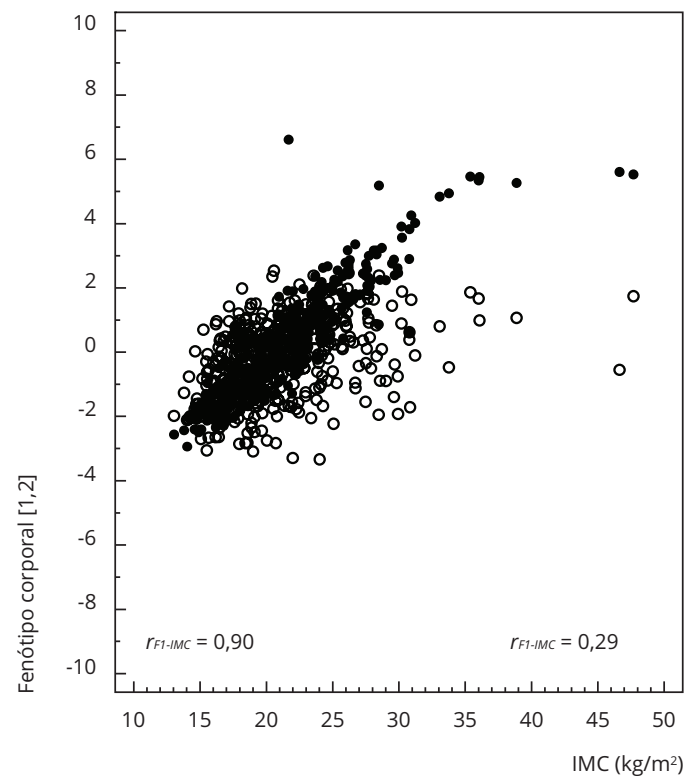

- F1 adiposidade

O F2puberdade

A associação entre a maturação sexual e o aumento da massa muscular já foi relatada anteriormente 1,7,38. No entanto, até onde pudemos buscar na literatura, não encontramos publicações que relatassem essas associações em análises multidimensionais, como a aqui apresentada. A multidimensionalidade permite que se possam discutir simultaneamente os vários aspectos envolvidos na composição corporal e na modificação dos caracteres sexuais secundários do indivíduo. Essa análise foi crucial para esclarecer as diferentes composições de massas corporais semelhantes observadas nos fenótipos 1 e 2, por exemplo.

$\mathrm{O} F 3_{\text {bioquímico }}$ e o $\mathrm{F} 4$ muscular representados por marcadores bioquímicos e variáveis da composição corporal mostram independência da maturação sexual. Os marcadores bioquímicos colesterol, triglicerídeos e glicose mostraram leve associação com circunferência da cintura e com IMC, por outro lado, não apresentaram associação com as variáveis de maturação sexual 36,38,39. Esse resultado sugere não haver influência da maturação sexual sobre os marcadores bioquímicos a ponto de criar um novo parâmetro que os inclua como ajuste de diagnóstico do estado nutricional do adolescente. O acúmulo excessivo de massa corporal durante a maturação sexual seria o principal fator para as desordens metabólicas, como alterações no colesterol, hemoglobina e glicose, e não propriamente efeito da maturação sexual 39,40 .

A idade, que representa o vetor cronológico associado à altura, é marcada no F2 puberdade, evidenciando a sua associação com o crescimento físico acelerado na adolescência. O vetor biológico, representado por acelerações hormonais e fenômenos característicos do processo de maturação sexual, também se associa ao crescimento linear e ao $\mathrm{F} 2$ puberdade. As diferenças encontradas nos estágios de maturação sexual estão associadas à mudança da idade cronológica, o que sugere um ritmo marcado para a evolução da maturação sexual independentemente da idade de início do processo 41.

A carga fatorial da variável massa corporal nos dois primeiros fenótipos corporais ( $\mathrm{F} 1_{\text {adiposidade }}$ e F $2_{\text {puberdade }}$ ) apresenta relação com duas composições corporais diferentes: quando há expansão 


\section{Figura 2}

Correlação dos $F 2_{\text {puberdade }}$ e $3_{\text {bioquímicos’ }}$ definidos por análise de componentes principais (ACP) com o índice de massa corporal (IMC), segundo sexo. Piracicaba, São Paulo, Brasil, 2012.

2a) Masculino



2b) Feminino

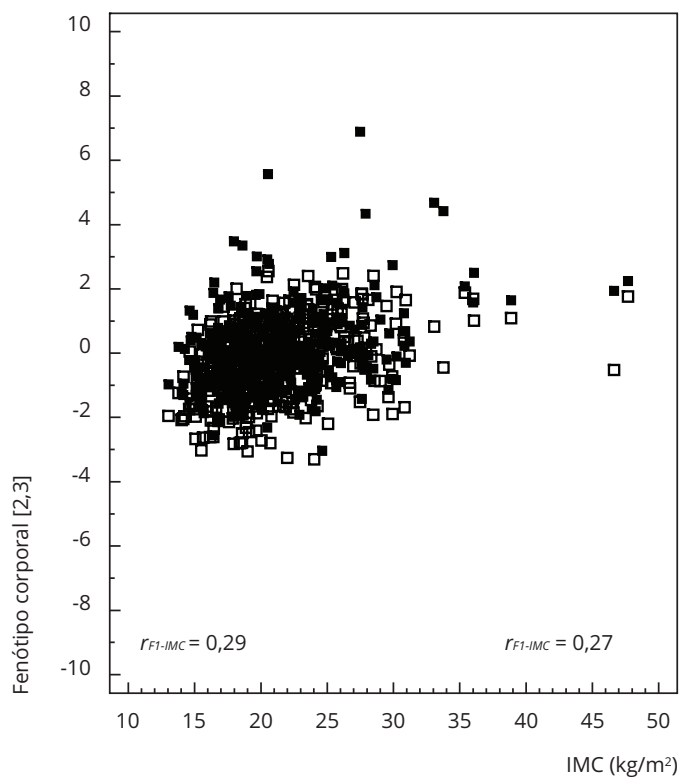

- F2puberdade

$\square$ F3bioquimicos

da massa e do volume corporal simultaneamente, esse processo está associado ao aumento da gor-

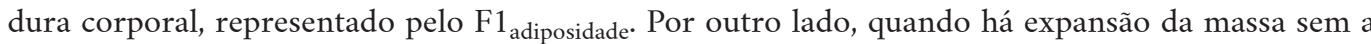
expansão do volume corporal, esse processo está associado ao crescimento físico linear, baseado no acúmulo de massa magra (F2 puberdade ${ }^{35,39}$. Desta forma, a massa corporal, quando expressa pelo IMC isoladamente, torna-se um potencial fator de confusão, já que não permite a diferença entre os dois fenômenos apresentados acima. Essa indiferenciação pode apresentar repercussões no processo de classificação do estado nutricional baseado na massa corporal nesse grupo etário.

Os estudos que são favoráveis ao ajuste do IMC pela maturação sexual diferem do presente estudo por incluírem o IMC na análise, e não massa corporal e altura separadamente. Neste estudo, consideramos altura e massa corporal variáveis distintas e com o mesmo grau de importância. Essa diferença pontual abre a possibilidade, que será explorada em análises futuras, de marcar o fenótipo associado à maturação sexual com base em variáveis antropométricas que o caracterizam, sem a necessidade de se avaliar especificamente os estágios de pelos pubianos e gônadas ou mamas. A favor dessa possibilidade, está o resultado de somente o $\mathrm{F} 1_{\text {adiposidade }}$ ter apresentado associação com o IMC.

O perfil de nossos resultados sugere que a análise isolada do IMC é indutora de alguns dos equívocos observados na avaliação do estado nutricional dos adolescentes durante a maturação sexual. A avaliação do estado nutricional baseada no IMC considera um recorte específico para o diagnóstico nutricional (massa corporal e altura). Já a análise multivariada permitiu que todas as variáveis utilizadas na avaliação do estado nutricional de adolescentes fossem interpretadas conjuntamente. A análise multivariada do estado nutricional possibilitou a descrição de fenótipos corporais distintos. Além disso, contribuiu para que pudéssemos olhar com um espectro mais apurado a verdadeira contribuição de cada variável nos fenótipos gerados e a distribuição das variáveis em cada fenótipo.

As limitações deste estudo foram: (a) informações autorreferidas para os dados de maturação sexual, o que pode ter acarretado omissão de informações corretas e dúvidas sobre o real estágio em 
Figura 3

Correlação dos $\mathrm{F} 2_{\text {puberdade }}$ e $\mathrm{F} 4_{\text {muscular, }}$ definidos por análise de componentes principais (ACP) com o índice de massa corporal (IMC), segundo sexo. Piracicaba, São Paulo, Brasil, 2012.

3a) Masculino

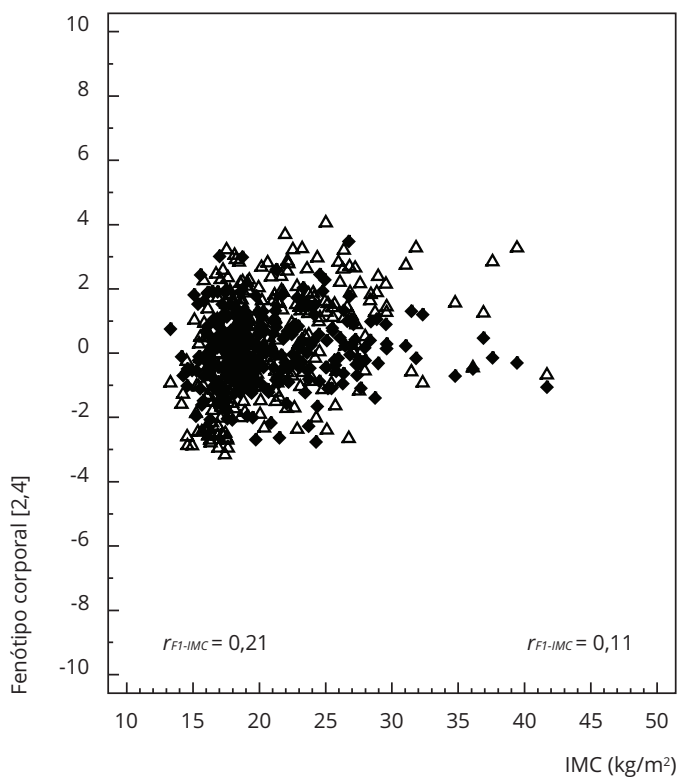

3b) Feminino

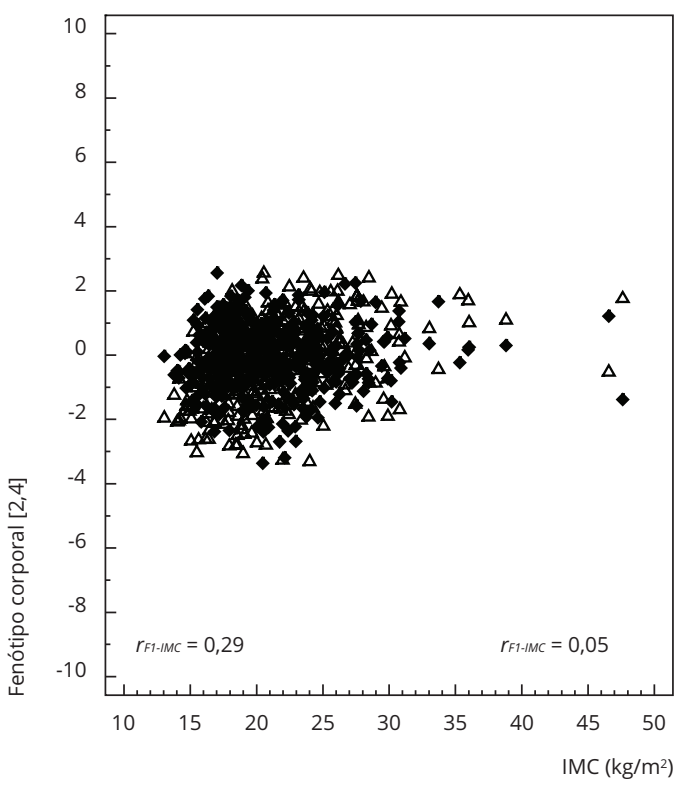

$\Delta$ F2puberdade

$\triangle \mathrm{F} 4$ muscular

que o adolescente se encontraria; e (b) cobertura da faixa etária, visto que o questionário de maturação sexual foi aplicado somente nos adolescentes de 10 a 15 anos, considerando que, nessa faixa etária, se concentram as maiores modificações causadas pelo processo da puberdade. Em futuros artigos, pretendemos realizar a mesma análise com dados de outros países, para verificar a replicabilidade dos resultados que obtivemos neste estudo.

\section{Conclusão}

O uso da análise multivariada para estimativa de fenótipos corporais em adolescentes púberes permitiu a descrição e a interpretação, em separado, dos processos de aumento preferencial de massa adiposa ou de massa magra implícitos no ganho de massa corporal nesse grupo etário. Essa distinção tem implicações diretas no processo de avaliação do estado nutricional. A presente abordagem está um passo à frente do uso de indicadores simples, já que analisa de forma ampla e integrada as medidas físicas, bioquímicas e da maturação sexual. Nossos resultados ressaltam a independência esperada entre os valores de antropometria, composição corporal, parâmetros bioquímicos e maturação sexual na análise do estado nutricional.

Nossos resultados apontam para a possibilidade de superar a dualidade entre a necessidade de ajustar previamente ou não, para maturação sexual, a classificação do estado nutricional baseado no IMC. A partir dos achados que apresentamos, torna-se possível imaginar um processo de avaliação clínica baseado em escores multivariados que integre a avaliação dos diferentes parâmetros com base em suas associações com variáveis antropométricas. Essa proposição está atualmente sendo explorada e será apresentada adiante. 


\section{Colaboradores}

J. Cumpian-Silva contribuiu com a concepção do estudo e a análise e interpretação dos dados e é responsável por todos os aspectos do trabalho na garantia da exatidão e integridade de qualquer parte da obra. W. L. Conde contribuiu com a concepção do estudo e a análise e interpretação dos dados e aprovou a versão final a ser publicada. A. E. M. Rinaldi e C. M. S. Mazzeti contribuíram com a redação do artigo e revisão crítica relevante do conteúdo intelectual.

\section{Agradecimentos}

J. Cumpian-Silva J e C. M. S. Mazzeti foram apoiadas pelo Conselho Nacional de Desenvolvimento Científico e Tecnológico (CNPq) e pela Coordenação de Aperfeiçoamento de Pessoal de Nível Superior (Capes), respectivamente, a quem agradecemos. Os dados foram coletados com apoio da Fundação de Amparo à Pesquisa do Estado de São Paulo (Fapesp), projeto Determinantes do Risco de Obesidade entre Adolescentes a Partir de Inquérito de Escolares com Amostra Mista: Transversal e Longitudinal (sob o processo no 2012/01283-3).

\section{Referências}

1. Cole TJ, Bellizzi MC, Flegal KM, Dietz WH Establishing a standard definition for child overweight and obesity world wide: international survey. BMJ 2000; 320:1240.

2. Vitolo MR, Campagnolo PDB, Barros ME, Gama CM, Ancona Lopez F. Evaluation of two classifications for overweight among Brazilian adolescents. Rev Saúde Pública 2007; 41:6536.

3. Barbosa KBF, Franceschini SCC, Priore SE Influência dos estágios de maturação sexual no estado nutricional, antropometria e composição corporal de adolescentes. Rev Bras Saúde Matern Infant 2006; 6:375-82.

4. Wang Y. Is obesity associated with early sexual maturation? A comparison of the association in American boys versus girls. Pediatrics 2002; 110:903-10.

5. Bini V, Celi F, Berioli MG, Bacosi ML, Stella $\mathrm{P}$, Giglio P, et al. Body mass index in children and adolescents according to age and pubertal stage. Eur J Clin Nutr 2000; 54:214-8.

6. Onis M, Onyango AW, Borghi E, Siyam A, Nishida C, Siekmann J. Development of a WHO growth reference for school-aged children and adolescents. Bull World Health Organ 2007; 85:660-7.

7. Cole TJ, Lobstein T. Extended international (IOTF) body mass index cut-offs for thinness, overweight and obesity. Pediatr Obes 2012; 7:284-94.

8. Gould SJ. Geometric similarity in allometric growth: a contribution to the problem of scaling in the evolution of size. Am Nat 1971; 105:113-36

9. Günther B, Morgado E. Theory of biological similarity revisited. J Theor Biol 1982; 96:54359.

10. Mul D, Fredriks AM, Buuren VS, Oostdijk W, Verloove-Venhorick SP, Wit JM. Pubertal development in The Netherlands 1965-1997. Pediatr Res 2001; 50:479-86.

11. Talma H, Schonbeck Y, Dommelen PV, Bakker B, Buuren SVM, HiraSing A. Trends in menarcheal age between 1955 and 2009 in the Netherlands. PLoS One 2013; 8:e60056.

12. Fredriks AM, Buuren SV, Burgmeijer RJ Meulmeester JF, Beuker RJ, Brugman E, et al. Continuing positive secular growth change in The Netherlands 1955-1997. Pediatr Res 2000; 47:316-23.

13. Fredriks AM, Buuren SV, Jeurissen SE, Dekker FW, Verloove-Vanhorick SP, Wit JM. Height, weight, body mass index and pubertal development reference values for children of Turkish origin in the Netherlands. Eur J Pediatr 2003; 162:788-93.

14. Parent AS, Teilmann G, Juul A, Skakkebaek NE, Toppari J, Bourguignon JP. The timing of normal puberty and the age limits of sexual precocity: variations around the world, secular trends, and changes after migration. Endocr Rev 2003; 24:668-93. 
15. Gage TB. The evolution of human phenotypic plasticity: age and nutritional status at maturity. Hum Biol 2003; 75:521-37.

16. Peres SV, Latorre MRDO, Slater B, Tanaka LF, Silva MV. Prevalência de excesso de peso e seus fatores associados em adolescentes da rede de ensino público de Piracicaba, São Paulo. Rev Paul Pediatr 2012; 30:57-64.

17. Romero A, Slater B, Florindo AA, Latorre MRDO, Cezar C, Silva MV. Determinantes do índice de massa corporal em adolescentes de escolas públicas de Piracicaba, São Paulo. Ciênc Saúde Coletiva 2014; 15:141-9.

18. Lohman TG, Going SB. Body composition assessment for development of an international growth standard for preadolescent and adolescent children. Food Nutr Bull 2006; 27(4 Suppl 5):S314-25.

19. Lohman TG, Roche AF, Martorell R. Anthropometric standardization reference manual. Champaign: Human Kinetics Books; 1988.

20. International Obesity Task Force. EU platform on diet, physical activity and health. http:// ec.europa.eu/health/ph_determinants/life_ style/nutrition/documents/iotf_en.pdf (acessado em 15/Set/2010).

21. Dittmar M. Reliability and variability of bioimpedance measures in normal adults: effects of age, gender and body mass. Am J Phys Anthropol 2003; 122:361-70.

22. Fernandes RA, Rosa CS, Buonani C, Oliveira AR, Freitas Júnior IF. The use of bioelectrical impedance to detect excess visceral and subcutaneous fat. J Pediatr 2007; 83:529-34.

23. Marshall WA, Tanner JM. Variations in pattern of pubertal changes in girls. Arch Dis Child 1969; 44:291-303.

24. Marshall WA, Tanner JM. Variations in pattern of pubertal changes in boys. Arch Dis Child 1970; 45:13-23.

25. Ministério da Saúde. Caderneta de saúde do adolescente. 2a Ed. Brasília: Ministério da Saúde; 2012. (Série F. Comunicação e Educação em Saúde).

26. Chan NP, Sung RY, Kong AP, Goggins WB, So HK, Nelson EA. Reliability of pubertal selfassessment in Hong Kong Chinese children. J Paediatr Child Health 2008; 44:353-8.

27. World Health Organization. Nutrition in adolescence - issues and challenges for the health sector: issues in adolescent health and development. Geneva: World Health Organization; 2005.
28. Faria ER, Franceschini SCC, Peluzio MCG, Sant'Ana LFR, Priore SE. Aspectos metodológicos e éticos da avaliação da maturação sexual de adolescentes. Rev Paul Pediatr 2013; 31:398-405.

29. Martin RHC, Uezu R, Parra SA, Arena SS, Bojikian LP, Bohme MTS. Auto-avaliação da maturação sexual masculina por meio da utilização de desenhos e fotos. Rev Paul Educ Fís 2001; 15:212-22.

30. Ministério da Saúde. Técnicas para coleta de sangue. 3a Ed. Brasília: Ministério da Saúde; 2001.

31. Sociedade Brasileira de Cardiologia. I diretriz de prevenção da aterosclerose na infância e na adolescência. Arq Bras Cardiol 2005; 85 Suppl 6:3-36.

32. American Diabetes Association. Standards of medical care in diabetes. Diabetes Care 2005; 28 Suppl 1:4-36.

33. Jolliffe IT. Principal component analysis. 2nd Ed. New York: Springer-Verlag; 2002.

34. Joliffe IT, Morgan BJ. Principal component analysis and exploratory factor analysis. Stat Methods Med Res 1992; 1:69-95.

35. Daniels SR, Khoury PR, Morrison JA. The utility of body mass index as a measure of body fatness in children and adolescents: differences by race and gender. Pediatrics 1997; 99:804-7.

36. Kaplowitz PB. Link between body fat and the timing of puberty. Pediatrics 2008; 121:208-17.

37. Morrison JA, Barton BA, Biro FM, Sprecher DL. Sex hormones and the changes in adolescent male lipids: longitudinal studies in a biracial cohort. J Pediatr 2003; 142:637-42.

38. Fukunaga Y, Takai Y, Yoshimoto T, Fujita E, Yamamoto M, Kanehisa H. Effect of maturation on muscle quality of the lower limb muscles in adolescent boys. J Physiol Anthropol 2015; 33:30.

39. Duarte MFS. Maturação física: uma revisão da literatura, com especial atenção à criança brasileira. Cad Saúde Pública 1993; 9:71-84.

40. Serrano HMS, Carvalho GQ, Pereira PF, Peluzio MCG, Franceschini SCC, Priore SE. Composição corpórea, alterações bioquímicas e clínicas de adolescentes com excesso de adiposidade. Arq Bras Cardiol 2010; 95:464-72.

41. van Buuren S, Ooms JC. Stage line diagram: an age-conditional reference diagram for tracking development. Stat Med 2009; 28:1569-79. 


\section{Abstract}

The study aims to describe body phenotypes estimated by multivariate technique for the assessment of nutritional status in adolescence during the process of sexual maturation. The sample consisted of 833 schoolchildren 10 to 15 years of age, selected by complex sampling in Piracicaba, São Paulo State, Brazil. Body phenotypes were defined by principal components analysis (PCA) based on anthropometric data (body mass, height, skinfolds, and waist circumference), body composition (phase angle measurement by bioelectric impedance), biochemical parameters (triglycerides, glucose, total/ LDL cholesterol ratio, hemoglobin), and sexual maturation (self-classification of stages of pubic hair and breasts or genitals). Body phenotypes were: $P 1_{\text {adiposity }}$ characterized by a positive association with the variables skinfolds, body mass, and waist circumference; $P 2_{\text {puberty }}$, characterized by positive association with stages of pubic hair and breasts in girls or genitals in boys, height, and age; $P 3_{\text {biochemical }}$ characterized by positive association with cholesterol, triglycerides, and glucose; and $P 4_{\text {muscular }}$ characterized by positive association with phase angle and hemoglobin and negative association with glucose. Only $P 1_{\text {adiposity }}$ showed a strong association with body mass index, and there was independence between $P 1_{\text {adiposity }}$ and $P 2_{\text {puberty }}$. Our results highlight the independence between biochemical parameters, anthropometry, body composition, and sexual maturation. Body phenotypes can provide the basis for calculating scores on likelihood of obesity based on anthropometric variables and overcoming ambiguity with the use of body mass.

Sexual Maturation; Biomarkers; Body Composition; Adolescent

\section{Resumen}

Nuestro objetivo es describir fenotipos corporales estimados por técnica multivariada para la evaluación del estado nutricional en la adolescencia, durante el proceso de madurez sexual. La muestra estaba compuesta por 833 adolescentes, escolares de 10 a 15 años, seleccionados por una muestra compleja en Piracicaba, São Paulo, Brasil. Los fenotipos corporales fueron definidos por un análisis de componentes principales (ACP), a partir de datos antropométricos (masa corporal, altura, pliegues cutáneos y circunferencia de la cintura), de composición corporal (ángulo de fase medido por impedancia bioeléctrica), bioquímicos (triglicéridos, glucosa, razón colesterol total/LDL, hemoglobina) y de madurez sexual (autoclasificación de las etapas de pelos púbicos y mamas o gónadas). Los fenotipos corporales fueron: $F 1_{\text {adiposidad }}$ caracterizado por la asociación positiva con las variables pliegues cutáneos, masa corporal y circunferencia de la cintura; $F 2_{\text {pubertad }}$ caracterizado por la asociación positiva con etapas de pelos púbicos, mama en niñas o gónadas en niños, altura y edad; $F 3_{\text {bioquimico, caracterizado por la asociación po- }}$ sitiva con colesterol, triglicéridos y glucosa; y $F 4_{\text {muscular }}$ caracterizado por la asociación positiva con ángulo de fase, hemoglobina y negativa con glucosa. Solamente $F 1_{\text {adiposidad }}$ presenta una asociación fuerte con el índice de masa corporal, $y$ existe independencia entre $F 1_{\text {adiposidad }}$ y $F 2_{\text {pubertad }}$. Nuestros resultados resaltan la independencia presentada entre parámetros bioquímicos, de antropometría, de composición corporal y de madurez sexual. La propuesta de fenotipos corporales podrá servir de base para el cálculo de marcadores de probabilidad de estar obeso, a partir de las variables antropométricas, $y$ superar así la ambigüedad en la utilización de la masa corporal.

Maduración Sexual; Biomarcadores;

Composición Corporal; Adolescente
Recebido em 04/Abr/2017

Versão final reapresentada em 08/Set/2017 Aprovado em 02/Out/2017 